\title{
434 - Treatment Patterns and Needs for Dementia-related Psychosis Described by Patients and Care Partners (Caregivers): an Observational, Prospective Study to Describe the Patient Experience
}

Authors: Teresa Brandt ${ }^{1}$, Theresa Frangiosa ${ }^{2}$, Virginia Biggar ${ }^{2}$, Angela Taylor ${ }^{3}$, Bill Keller ${ }^{1}$, Vic Abler $^{1}$

${ }^{1}$ ACADIA Pharmaceuticals Inc., San Diego, CA, USA

${ }^{2}$ UsAgainstAlzheimer's (UsAA), Washington DC, USA

${ }^{3}$ Lewy Body Dementia Association, Lilburn, GA, USA

\begin{abstract}
Background: We aimed to describe current treatment patterns and unmet needs of persons with DRP from a patient and care partner (CP) perspective.

Methods: This observational, non-interventional, prospective study used a mixed-methods approach with qualitative interviews and a quantitative online survey. Persons with DRP and CP of persons with DRP reported the effectiveness of current treatments using a visual analog scale (VAS) of 0 ("not at all well") to 5 ("extremely well") and ranked benefits of an ideal treatment. CP burden was outside the scope of this study.
\end{abstract}

Results: The qualitative interview was completed by 1 patient and $15 \mathrm{CP}$. Current treatments included atypical antipsychotics, antidepressants, anxiolytics, and benzodiazepines. Participants commonly indicated a need to improve patients' ability to know what is real vs not real.

The online survey was completed by 26 patients and $186 \mathrm{CP}$ on behalf of patients under their care. Common treatments used included atypical antipsychotics (27\% of patients, $41 \%$ of CP) and psychological/ behavioral therapy (23\% of patients, $8 \%$ of CP). Many participants reported no current treatment ( $42 \%$ of patients, $42 \%$ of CP). Participants reported that current treatment methods were less than moderately helpful in treating patients' most impactful symptoms with a median VAS score of 2.0/5 reported by patients and CP (mean VAS score [standard deviation] = 2.33 [1.0] for patients [ $n=15$ ], mean [standard deviation] VAS score $=2.4$ [1.3] for CP [n=104]). Discontinuation of a treatment was reported by 11 patients and $115 \mathrm{CP}$, most commonly due to a side effect ( $27 \%$ of patients, $31 \%$ of $\mathrm{CP}$ ), doctor's recommendation ( $27 \%$ of patients, $14 \%$ of CP), or lack of symptom improvement ( $9 \%$ of patients, $28 \%$ of CP). Participants ranked the ability to distinguish what is real vs not real ( $35 \%$ of patients, $49 \%$ of CP) and overall symptom improvement ( $42 \%$ of patients, $23 \%$ of $\mathrm{CP}$ ) as the most important benefits of an ideal treatment.

Conclusions: Patients and CPs reported either not taking any DRP treatments or that current treatments were associated with side effects along with limited efficacy. There is an unmet need for safe and effective treatments for DRP.

Study Sponsored By: ACADIA Pharmaceuticals Inc.

\section{Disclosures}

TF is a consultant with Frangiosa \& Associates, LLC.

$\mathrm{VB}$ and $\mathrm{AT}$ have no relevant financial relationships to disclose.

TB, BK, and VA are employees of and may hold stock and/or stock options with ACADIA Pharmaceuticals Inc. 